\title{
Making a commercial atomic force microscope more accurate and faster using positive position feedback control
}

\author{
I. A. Mahmood and S. O. Reza Moheimani ${ }^{\text {a) }}$ \\ School of Electrical Engineering and Computer Science, The University of Newcastle, Callaghan, New South \\ Wales 2308, Australia
}

(Received 29 January 2009; accepted 28 May 2009; published online 23 June 2009)

\begin{abstract}
This paper presents experimental implementation of a positive position feedback (PPF) control scheme for vibration and cross-coupling compensation of a piezoelectric tube scanner in a commercial atomic force microscope (AFM). The AFM is a device capable of generating images with extremely high resolutions down to the atomic level. It is also being used in applications that involve manipulation of matter at a nanoscale. Early AFMs were operated in open loop. Consequently, they were susceptible to piezoelectric creep, thermal drift, hysteresis nonlinearity, and scan-induced vibration. These effects tend to distort the generated image and slow down the scanning speed of the device. Recently, a new generation of AFMs has emerged that utilizes position sensors to measure displacements of the scanner in three dimensions. These AFMs are equipped with feedback control loops that work to minimize the adverse effects of hysteresis, piezoelectric creep, and thermal drift on the obtained image using proportional-plus-integral (PI) controllers. These feedback controllers are often not designed to deal with the highly resonant nature of an AFM's scanner nor with the cross coupling between various axes. In this paper we illustrate the improvement in accuracy and imaging speed that can be achieved by using a properly designed feedback controller such as a PPF controller. Such controllers can be incorporated into most modern AFMs with minimal effort since they can be implemented in software with the existing hardware. Experimental results show that by implementing the PPF control scheme, relatively good images in comparison with a well-tuned PI controller can still be obtained up to line scan of $60 \mathrm{~Hz}$. (C) 2009 American Institute of Physics. [DOI: 10.1063/1.3155790]
\end{abstract}

\section{INTRODUCTION}

The atomic force microscope $(\mathrm{AFM})^{1,2}$ was conceived to generate three-dimensional (3D) images of material surfaces with very high accuracies. As illustrated in Fig. 1 in this device, a microcantilever with a very sharp tip is scanned over a sample at distances of the order of a few nanometers or less. Interatomic forces between the tip and the sample force the microcantilever to deflect. This deflection is measured by a laser and a photodetector. The force experienced by the microcantilever is a nonlinear function of the tipsample separation. ${ }^{2}$ This enables the AFM to operate in a number of modes. In contact mode, illustrated in Fig. 1, the tip experiences a repulsive force, which is kept constant during the scan by a feedback loop. The controller is often a proportional-plus-integral (PI) compensator. In this mode of operation, the tip exerts a relatively large normal force and a considerable lateral force on the sample. Consequently, the probe is subject to significant wear. Hence, this mode may not be suitable for soft samples that can be damaged easily, e.g., biological samples.

A critical component of an AFM is its scanning head that moves either the sample or the probe in a raster pattern in the $x-y$ plane. It also controls the distance between the probe and the sample through the $z$-axis servo. The use of a piezoelec-

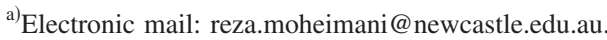

tric tube actuator with quartered external electrodes for 3D positioning was first proposed in Ref. 3. Today, the majority of commercially available AFMs use a piezoelectric tube scanner for $x-y-z$ positioning, although flexure-based nanopositioners ${ }^{4-6}$ are emerging as a viable albeit more expensive alternative. Accuracy and speed of operation of an AFM are adversely affected by a number of properties inherent to piezoelectric materials.

Piezoelectric actuators exhibit hysteretic behavior. When a triangular voltage signal is applied to a piezoelectric actuator, the resulting displacement can deviate from linear by as much as $15 \%$ between the forward and backward movements. Earlier AFMs compensate for hysteresis effect by perturbing the input triangular signal to achieve an acceptable trajectory, thus minimizing the effect of this particular form of nonlinearity. ${ }^{2}$ Modeling a piezoelectric actuator as a linear dynamic system cascaded with a static nonlinearity and then compensating for the nonlinearity through inversion is another approach that has been researched. ${ }^{7-9}$ However, the authors are not aware if it has been incorporated into an existing AFM.

Another undesirable property of piezoelectric materials is creep. When the applied voltage to a piezoelectric actuator undergoes a sudden change, the piezoelectric material will creep. This can result in significant loss in precision when positioning is required over extended periods of time. ${ }^{10} \mathrm{In}$ particular during slow operation of AFMs, creep can result in 


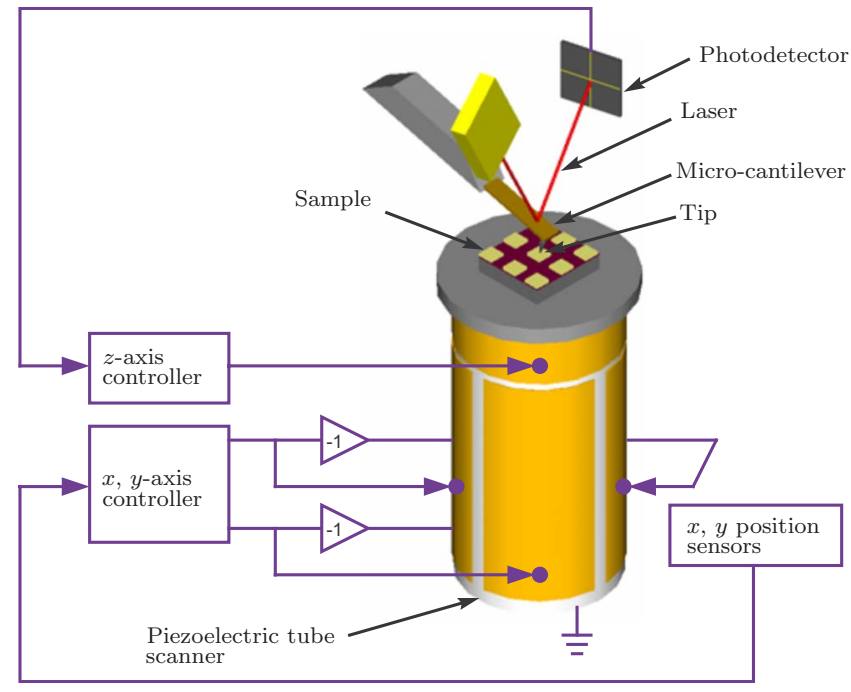

FIG. 1. (Color online) Basic AFM schematic with feedback controllers.

significant distortions in the generated image. ${ }^{11}$ Creep exacerbates the effect of hysteresis at the turning point of the scanning trajectory and it has an adverse effect on the vertical positioning of the sample. While a number of methods have been proposed to deal with this phenomenon, ${ }^{12-14}$ the most widely used approach in earlier AFMs has been to allow sufficient time for the effect of creep to disappear.

Yet another troublesome feature of AFMs particularly in applications that involve nanoscale manipulation of materials is the thermal drift of their mechanical setup. This arises from the thermal expansion and contraction of their mechanical components. In a typical AFM operated in ambient temperature, a $1^{\circ}$ change in temperature has been shown to cause as much as $50 \mathrm{~nm}$ drift. ${ }^{15}$ This effect can be suppressed in low temperature experiments, e.g., if the AFM is operated in ultrahigh vacuum. However, in applications which involve interrogation or manipulation of the matter in ambient temperature it may amount to a substantial hurdle. ${ }^{15}$

As indicated above, feedback has always been an integral part of every AFM for vertical positioning. A feedback controller requires a measurement signal to operate effectively. In an AFM, this measurement is conveniently made available by the photodetector, enabling accurate vertical positioning of the scanner. The utilization of feedback to improve lateral positioning of the scanner, however, requires displacement sensors to be incorporated into the device. These sensors were not included in scanners of earlier AFMs. However, they are progressively being built into a new generation of commercially available AFMs. The ability to use feedback for lateral positioning brings about a number of exciting possibilities some of which have already materialized in commercially available AFMs. For example, PI controllers have been used to reduce the effects of hysteresis, creep, and thermal drift with significant success. Apart from the above complicating factors, there are two other issues that hamper the operation of an AFM: (i) the highly resonant nature of the scanner and (ii) the cross coupling between the various axes of a piezoelectric tube. ${ }^{16}$

To force the free end of the tube to move in a raster pattern, a triangular signal is applied to the $x$-axis (fast axis) electrodes and a slowly increasing staircase signal is applied to the $y$-axis (slow axis) electrodes. A triangular waveform contains all odd harmonics of the fundamental frequency. The amplitudes of these harmonic signals attenuate as $1 / n^{2}$, with $n$ being the harmonic number. ${ }^{17}$ If a fast triangular waveform is applied to the scanner, there are high chances that one of the higher amplitude harmonic signals will excite the resonance. Consequently, instead of following a perfect triangle, the free end of the tube traces a distorted triangular waveform along the $x$ axis. This can significantly distort the image. To avoid this complication, the scanning speed of AFMs is often limited to about $1 \%$ of the scanner's first resonance frequency. A widely used approach to deal with this issue, during fast scans, is to shape the tracking signal such that it does not excite the tube's resonance. ${ }^{14,18}$ A downside of this approach is that the tube is still mechanically very lightly damped, and thus susceptible to external disturbances and noise. The existence of cross coupling between the various axes of an AFM scanner can substantially distort the image. ${ }^{16,19}$ It can also be a major problem in applications that involve manipulation of matter at nanoscale.

The use of feedback control techniques other than PI controllers to improve accuracy and speed of AFMs has been undertaken by a number of researchers for the past decade. One of the earliest attempts is reported in Ref. 20 where a noncontacting inductive sensor was used in measuring the lateral displacement of an AFM piezoelectric tube scanner. Lag-lead and $H_{\infty}$ controllers were designed and implemented on the tube, and the feasibility of reducing the adverse effects of creep and hysteresis were demonstrated. The authors also reported that the $H_{\infty}$ controller achieved damping of high-frequency vibrations. In Ref. 21, loop shaping techniques were used in designing feedback controller for a piezoelectric tube scanner equipped with optical displacement sensors to track a raster pattern. The closed-loop system was implemented on an AFM and their results show accurate positioning with a high degree of repeatability is achievable with the use of feedback control. In Ref. 22, a proportionalplus-derivative high-gain feedback controller and a feedforward input were used to compensate for creep, hysteresis, and vibration effects in an AFM piezoactuator system. The high-gain feedback controller was first used to linearize the piezoactuator by compensating for the creep and hysteresis. Then, the linearized piezoactuator was modeled to determine the feedforward input to account for the vibration effects. Their results indicated that the use of feedforward input reduces the tracking error more as compared to using only feedback control. Other examples of successful applications of feedback control techniques include Refs. 4, 23, and 24. A comprehensive review of the field can be found in Ref. 25.

In this work, we improve the performance of a commercial AFM by using a positive position feedback (PPF) control scheme to compensate the vibration and cross couplings of its piezoelectric tube scanner. At present, the focus of this work is only limited to compensating the vibration and cross couplings in the lateral axes of the scanner. The performance of the PPF control scheme is assessed by comparing surface topography images of a calibration grating obtained using this control scheme with the one obtained using a well-tuned 


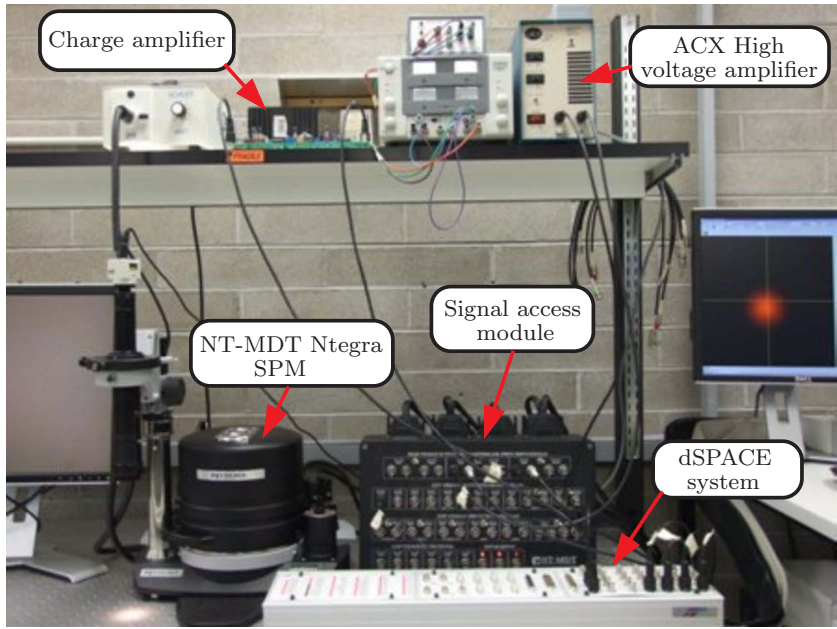

FIG. 2. (Color online) SPM system and experimental setup used in this work.

PI controller in the feedback loop. The remainder of the paper is arranged as follows. Section II provides descriptions of the AFM and other experimental setup used in this work. Modeling and identification of the system transfer functions are presented in Sec. III. Control schemes for the AFM scanner are devised in Sec. IV. In Sec. V, experimental results are presented to illustrate the drastic improvement in accuracy and imaging speed that can be achieved with the proposed control schemes. Finally, Sec. VI concludes the paper.

\section{EXPERIMENTAL SETUP}

Our experimental setup consisted of a NT-MDT Ntegra scanning probe microscope (SPM) shown in Fig. 2 that was configured to operate as an AFM. The AFM was retrofitted with a homemade charge amplifier ${ }^{26}$ on the fast axis. The charge amplifier has a constant gain of $68 \mathrm{nC} / \mathrm{V}$. Charge drive significantly minimized the effect of hysteresis on this axis of the scanner. The slow axis was driven by an ACX high voltage amplifier with a constant gain of 15 . It is sufficient to use the voltage amplifier because the effect of hysteresis on the slow axis is relatively small. The use of high feedback gain at low frequencies can minimize the effect of hysteresis effectively. A dSPACE-1103 rapid prototyping system was used to implement the feedback controllers in real time. The amplifiers and the AFM were interfaced with the dSPACE system using a signal access module that allowed direct access to the scanner electrodes. This setup enabled us to directly control the lateral movements of the scanner. However, the vertical positioning was achieved using the AFM control software. In other words we replaced the rastering mechanism of the AFM with our own system.

The scanner is a NT-MDT Z50309cl that performs 3D positioning in the SPM. Capacitive displacement sensors are incorporated into the scanner apparatus to allow for direct measurements of the tube displacement in $x, y$, and $z$ directions. These capacitive sensors have a sensitivity of $6.33 \mu \mathrm{m} / \mathrm{V}$. The system consists of a piezoelectric tube scanner with quartered internal and external electrodes. Such electrode arrangement allows the scanner to be driven in a bridge configuration ${ }^{27}$ where the electrodes are wired in pair

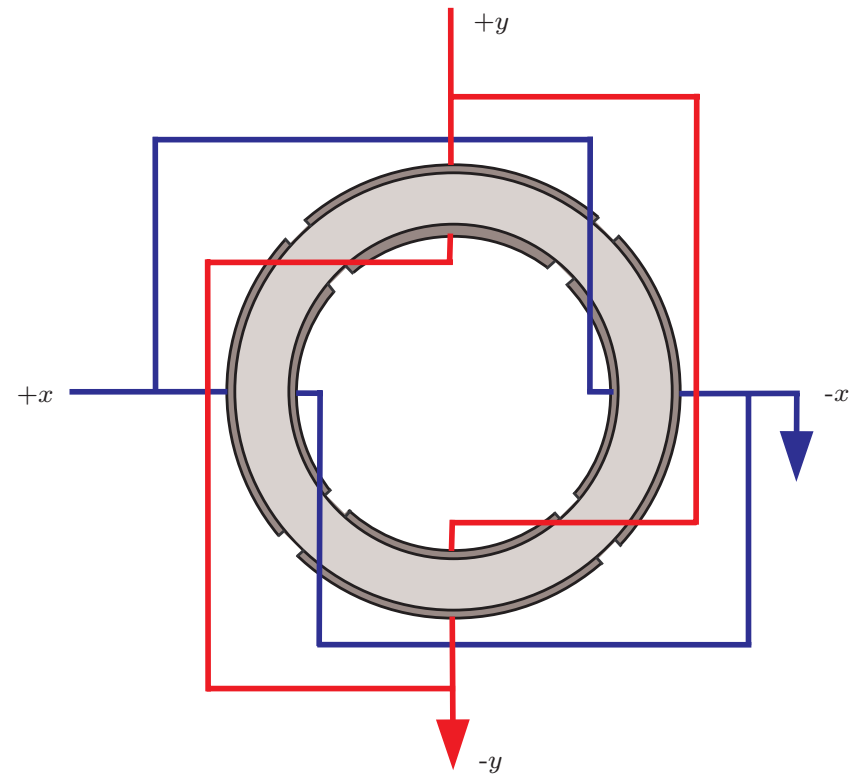

FIG. 3. (Color online) Top view of the piezoelectric tube with the internal and external electrode wired in a bridge configuration.

as illustrated in Fig. 3. These electrode pairs are referred to as $+x,-x,+y$, and $-y$ electrode pairs. An advantage of using the bridge configuration is that it halves the input voltage requirement as compared to the more well-known grounded internal electrode configuration. Nevertheless, in these experiments the $-x$ and $-y$ electrode pairs were grounded in order to simplify the experimental setup. Furthermore the need for large scanning range does not arise here since the scanner is only made to operate within $10 \%$ of the full lateral scanning range of the device.

A dSPACE rapid prototyping system equipped with CONTROLDESK software was interfaced to the AFM through an expansion box that allowed direct access to the capacitive sensor measurements and control signals that were to be applied to the piezoelectric actuator. During scans, measurements from the capacitive sensors and the photodiode were recorded and processed in MATLAB to generate AFM images.

\section{SYSTEM IDENTIFICATION}

In order to simplify the control design, the AFM scanner was treated as two single-input single-output systems. The inputs being the voltage signals applied to the charge amplifier $u_{x}$ and to the voltage amplifier $u_{y}$. The outputs of the systems are the tube displacement measurements in $x$ direction $c_{x}$ and in $y$ direction $c_{y}$. Accurate models of the systems were obtained through an experimental approach to modeling (system identification). The following frequency response functions (FRFs) were obtained nonparametrically using a dual-channel HP35670A spectrum analyzer,

$$
G_{c_{x} u_{x}}(i \omega)=\frac{c_{x}(i \omega)}{u_{x}(i \omega)}
$$

and

$$
G_{c_{y} u_{y}}(i \omega)=\frac{c_{y}(i \omega)}{u_{y}(i \omega)} .
$$


(a)
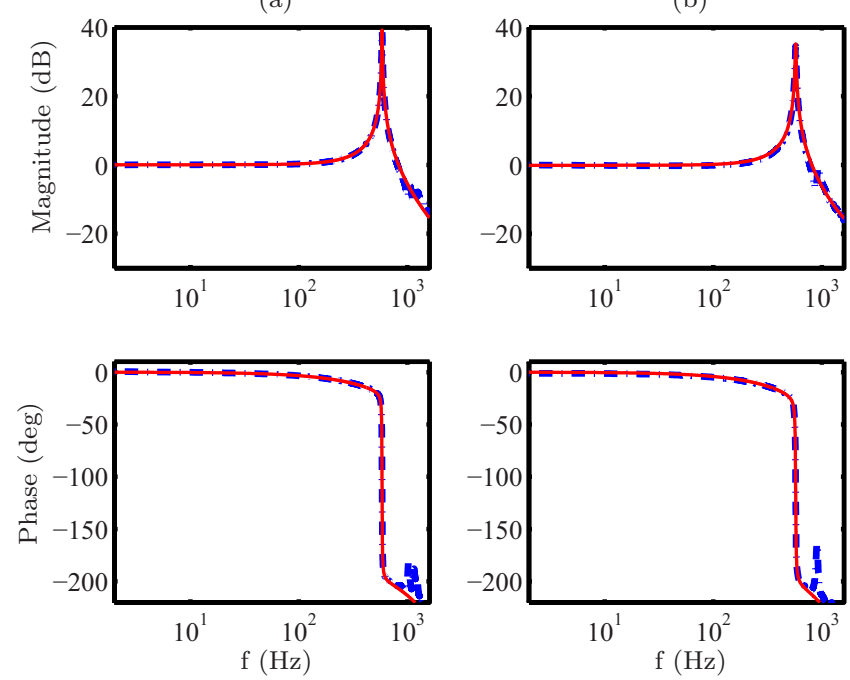

FIG. 4. (Color online) Experimental (dash-dot) and identified model (solid) frequency response of (a) $G_{c_{x} u_{x}}(i \omega)$ and (b) $G_{c_{y} u_{y}}(i \omega)$.

A band-limited random noise signal $(1-1600 \mathrm{~Hz})$ was generated using the spectrum analyzer and applied to the amplifiers as the input. The corresponding outputs from the capacitive displacement sensors were also recorded using the spectrum analyzer. These input-output data were processed to generate the FRF Eqs. (1) and (2) in a nonparametric form as illustrated in Fig. 4. Two second order models were fitted to the FRFs data using the frequency domain subspace-based system identification approach as described in Refs. 28 and 29. The following transfer functions were found to be a good fit as illustrated in Fig. 4:

$$
G_{c_{x} u_{x}}(s)=\frac{0.05311 s^{2}-1230 s+1.362 \times 10^{7}}{s^{2}+40.38 s+1.354 \times 10^{7}}
$$

and

$$
G_{c_{y} u_{y}}(s)=\frac{0.0849 s^{2}-1416 s+1.288 \times 10^{7}}{s^{2}+61.74 s+1.303 \times 10^{7}} .
$$

\section{CONTROLLER DESIGN}

To address the issues discussed in Sec. I, we designed feedback controllers to augment the damping of the scanner's transfer functions to achieve improved lateral positioning. Structure of the $x$-axis feedback controller is illustrated in Fig. 5. A similar controller was designed for the $y$ axis. In our experiments the fast axis was driven by a dc-accurate charge amplifier. Piezoelectric actuators are often driven by voltage amplifiers. Voltage-driven piezoelectric materials are

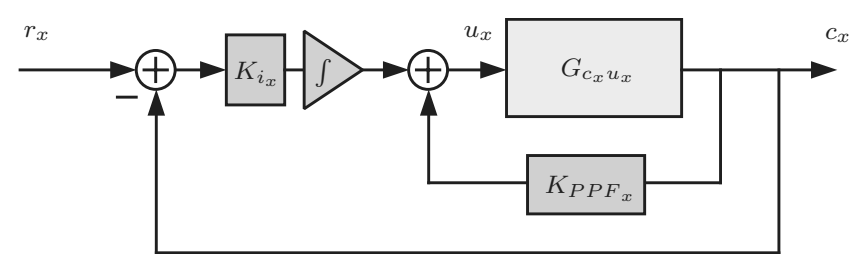

FIG. 5. Structure of the $x$ axis feedback controller. The inner feedback loop is a PPF controller designed to damp the highly resonant mode of the tube. Integral action is also incorporated to achieve satisfactory tracking. known to display hysteretic behavior. An interesting and unique property of piezoelectric materials is that when driven by a charge source, they display minimal hysteresis. This property has been known since 1980s. ${ }^{30,31}$ However, until very recently, it has been rarely used due to the difficulties associated with driving highly capacitive loads with commercially available charge or current amplifiers. Recently, we proposed ${ }^{26,32-34}$ a new construction for charge and current sources capable of regulating the dc profile of the actuator.

The overall control structure, illustrated in Fig. 5, consists of two feedback loops. The inner loop contains a PPF controller that works to increase the overall damping of the tube. PPF controllers were initially designed to suppress mechanical vibrations of highly resonant aerospace structures. ${ }^{35}$ They have been successfully implemented on a range of lightly damped structures. ${ }^{36-38}$ Their effectiveness in improving accuracy and bandwidth of nanopositioning systems was recently investigated in Ref. 23. PPF controllers have a number of important features. In particular, they have a simple structure, are easy to implement, and their transfer functions roll off at a rate of $40 \mathrm{~dB} /$ decade at higher frequencies. The latter is important in terms of the overall effect of the sensor noise on the scanner's positioning accuracy. The detail of the procedure that was followed to design these PPF controllers is documented in Ref. 23. The obtained PPF controllers can be described as

$$
K_{\mathrm{PPF}_{x}}(s)=\frac{9.242 \times 10^{6}}{s^{2}+6032 s+2.785 \times 10^{7}}
$$

and

$$
K_{\mathrm{PPF}_{y}}(s)=\frac{9.499 \times 10^{6}}{s^{2}+6062 s+2.753 \times 10^{7}} .
$$

The control system we designed also includes integral action as illustrated in Fig. 5. Inclusion of an integrator amounts to applying a high gain at low frequencies that reduces the effects of thermal drift, piezoelectric creep, and hysteresis to a minimum. An important benefit of the proposed combined feedback structure is the significant reduction that can be achieved in cross coupling between various axes of the scanner. The gain of the integrators were tuned to provide high closed-loop bandwidth but with reasonable gain and phase margin.

\section{EXPERIMENTAL RESULTS}

The performance of the PPF control scheme was first evaluated by measuring the closed-loop frequency responses of the system using the spectrum analyzer. In Fig. 6, the measured closed-loop frequency responses are plotted along with the open-loop frequency responses that were obtained in Sec. III. By inspecting Figs. 6(a) and 6(d) we observe that the closed-loop system bandwidth of both axes is about 300 Hz. Also, a damping of more than $30 \mathrm{~dB}$ at each resonant mode is apparent from the frequency responses. Note that with the current experimental setup, we were not able to measure the closed-loop frequency responses of the AFM scanner with the well-tuned PI controller that is built into the 

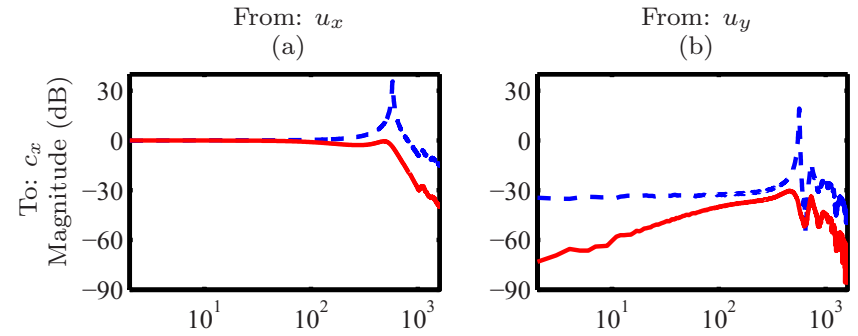

(c)
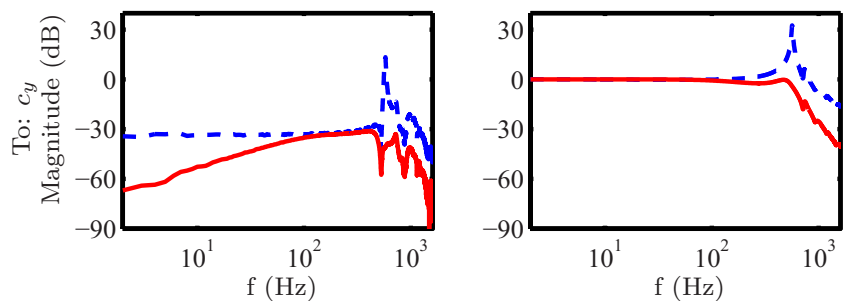

FIG. 6. (Color online) Open-loop (dash) and closed-loop (solid) frequency responses of the scanner. The resonant behavior of the scanner is improved by over $30 \mathrm{~dB}$ due to control action. The proposed feedback control strategy results in significant improvement in cross coupling between the fast and slow axes of the scanner.

AFM. When this controller is in use, access to the scanner electrodes through the signal access module and the raw signals obtained from the capacitive sensors are not made available to the user.

To appreciate the improvement achieved in lateral positioning of the scanner, we performed a simple experiment. In open loop, a $2 \mathrm{~Hz}$ triangular signal was applied to the $x$ axis of the piezoelectric tube to achieve an $8 \mu \mathrm{m}$ scan. The displacements in the $x$ and $y$ axes of the tube were measured using the built-in capacitive displacement sensors. A similar experiment was then carried out with the PPF control scheme implemented on the tube. The results are plotted in Fig. 7(a). A similar set of experiments were performed at $30 \mathrm{~Hz}$ to simulate a fast scan. The results are plotted in Fig. 7(c). A (a)

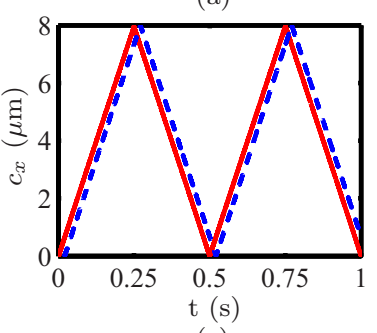

(c)

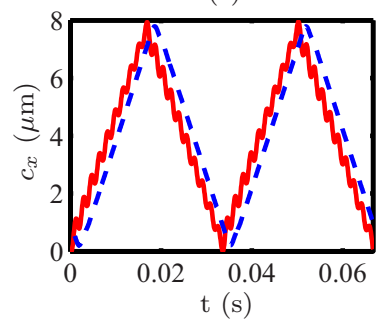

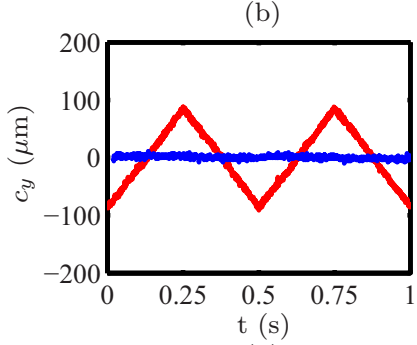

(d)

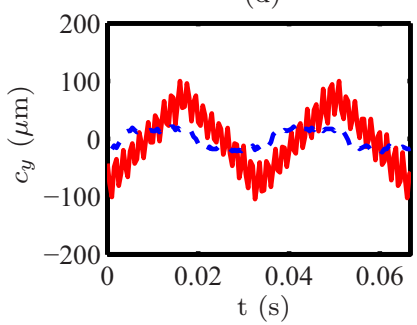

FIG. 7. (Color online) Closed-loop (dash) and open-loop (solid) tracking performance (left) and cross-coupling properties (right) of the scanner for 2 $\mathrm{Hz}$ scan (top) and $30 \mathrm{~Hz}$ scan (bottom). (A small phase shift was purposely added into the close-loop time responses in order to clearly display the open- and closed-loop time responses.) considerable improvement can be observed by comparing the open-loop and closed-loop motions of the tube. During a fast scan we can observe significant distortions due to the excitation of the tube's resonance. By applying the feedback controller, we managed to significantly reduce the distortions and achieved a considerable improvement in tracking performance. In particular, for the $30 \mathrm{~Hz}$ scan, the fast axis rms tracking error was reduced from 280 to $46 \mathrm{~nm}$, and the cross coupling to the slow axis was reduced from 50 to $16 \mathrm{~nm}$ due to control action. This improvement directly translates into an image with less distortion. A significant component of the closed-loop error is due to the phase shift between the desired and the achieved trajectories. This phase shift has a minimal effect on the image quality and can be handled using a feedforward controller.

The frequency responses for the cross-coupling terms of the AFM scanner were also obtained and illustrated in Figs. 6(b) and 6(c). In open loop, significant cross coupling can be observed between lateral axes of the scanner. For frequency ranges below the tube's resonance frequency, there is approximately $32 \mathrm{~dB}$ cross coupling between the $x$ and $y$ axes of the scanner. This means that an $8 \mu \mathrm{m}$ amplitude triangular motion of the $x$ axis will translate into approximately $0.2 \mu \mathrm{m}$ amplitude triangular motion of the $y$ axis and vice versa, generating substantial distortion in the resulting image. Also higher cross coupling exists at and close to the resonance frequency of the tube. The effect from the cross coupling can be observed in Figs. 7(b) and 7(d) where a triangular motion of approximately $0.18 \mu \mathrm{m}$ amplitude can be seen in the $y$ axis when the $x$ axis is made to produce an $8 \mu \mathrm{m}$ amplitude triangular motion. Note that at $30 \mathrm{~Hz}$ scan, in addition to the cross coupling, the distortions due to the excitation of the tube's resonance can also be observed in the $y$ axis.

In closed loop, Figs. 6(b) and 6(c) illustrate substantial decrease in the cross coupling between the lateral axes of the scanner. In particular, the cross coupling is less than $52 \mathrm{~dB}$ for low frequency ranges (i.e., $\leq 10 \mathrm{~Hz}$ ). This means that for a low frequency scan, an $8 \mu \mathrm{m}$ amplitude triangular motion of the $x$ axis will only translate into approximately $0.02 \mu \mathrm{m}$ amplitude triangular motion of the $y$ axis. Although there are no direct feedback controls on the cross-coupling terms, the inclusion of high-gain integrator in both axes has indirectly resulted in a significant reduction in the cross coupling. Nevertheless, the effect of the integral action diminishes as the frequency increases.

Having improved the lateral positioning of the scanner, we then moved on to investigate the overall improvement in imaging capability of our modified AFM. During the imaging, the AFM was operated in constant force mode using a microcantilever with spring constant of $0.2 \mathrm{~N} / \mathrm{m}$. The sample was a $20 \mathrm{~nm}$ feature-height NT-MDT TGQ1 calibration grating with a $3 \mu \mathrm{m}$ pitch. The well-tuned PI was first used to develop $8 \times 8 \mu \mathrm{m}^{2}$ images of the sample at 2,10 , and $30 \mathrm{~Hz}$ scan frequencies with a resolution of $256 \times 256$ pixels. Faster scans beyond $30 \mathrm{~Hz}$ with the AFM standard software, at this resolution, were not possible. In each case, a significant amount of time was devoted to tune the AFM so that the best possible image could be generated. We then imaged the 
(a) $f_{s}=2 \mathrm{~Hz}$

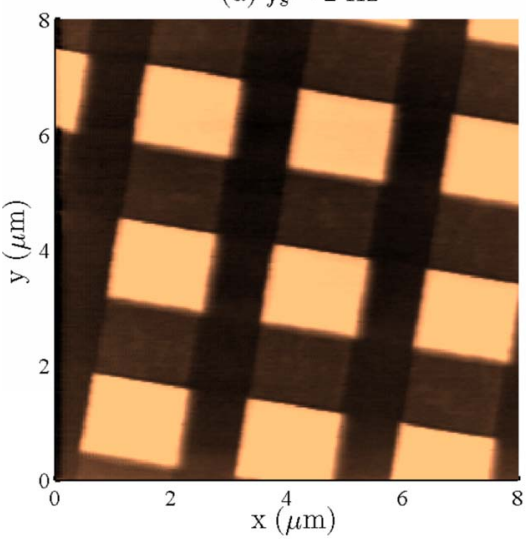

(b) $f_{s}=10 \mathrm{~Hz}$

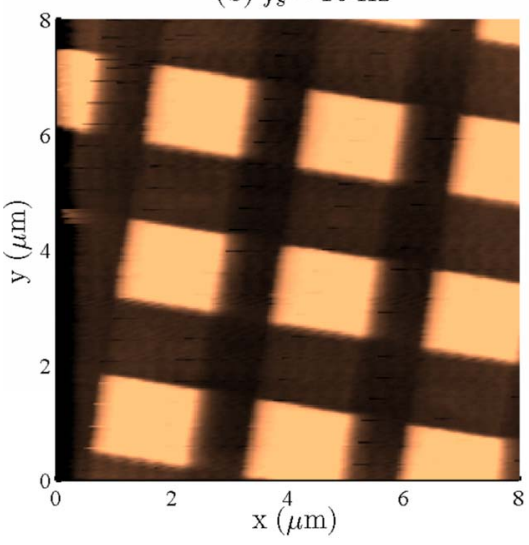

(c) $f_{s}=30 \mathrm{~Hz}$

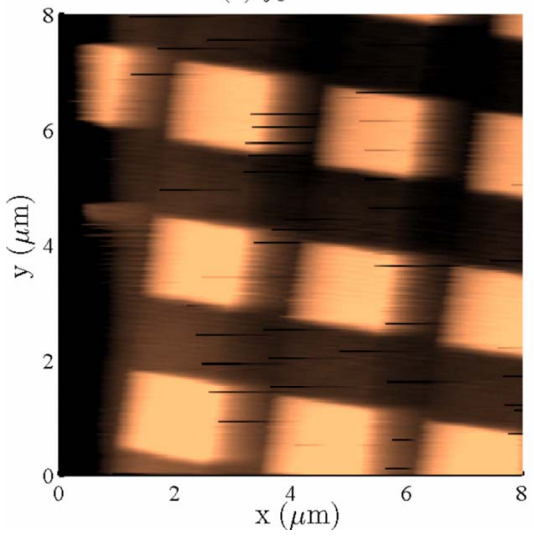

(d) $f_{s}=2 \mathrm{~Hz}$

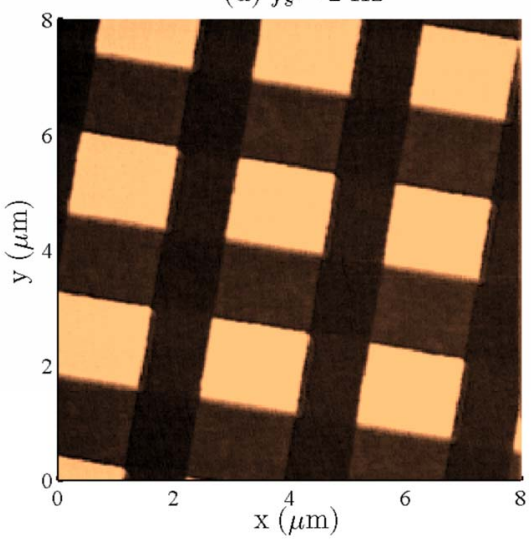

(e) $f_{s}=10 \mathrm{~Hz}$

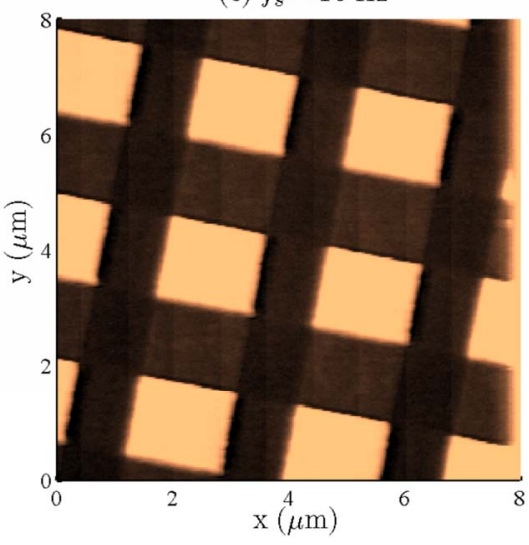

(f) $f_{s}=30 \mathrm{~Hz}$

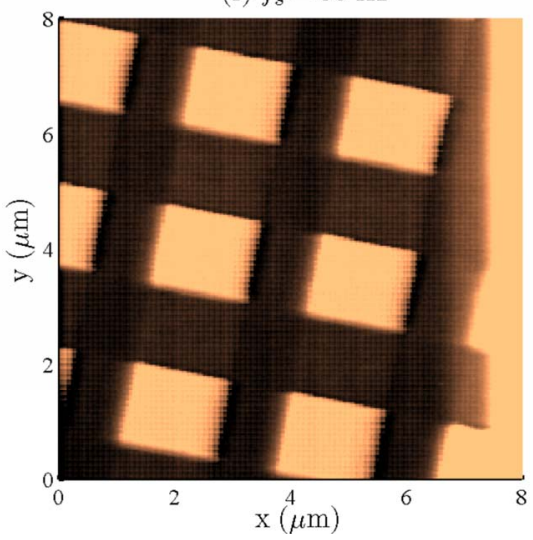

(g) $f_{s}=40 \mathrm{~Hz}$

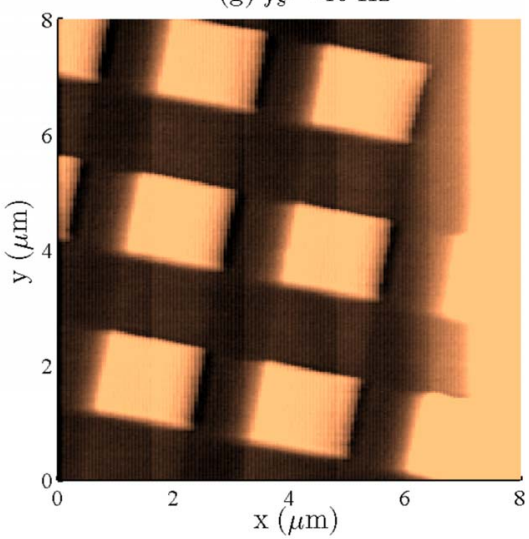

(h) $f_{s}=50 \mathrm{~Hz}$

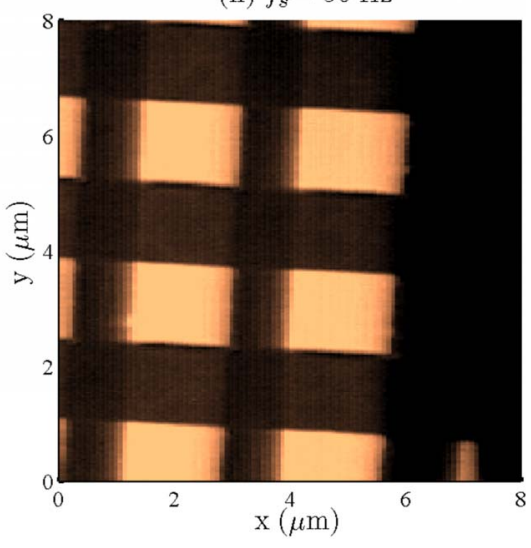

(i) $f_{s}=60 \mathrm{~Hz}$

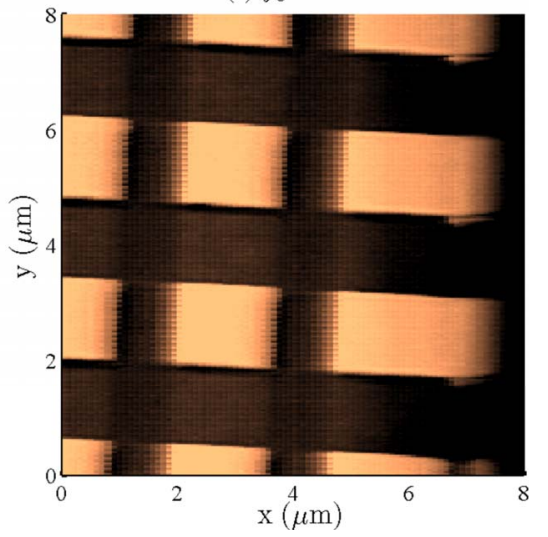

FIG. 8. (Color online) First two columns: AFM images of NT-MDT TGQ1 grating scanned in contact mode constant force at 2, 10, and 30 Hz. Images displayed in (a), (b), and (c) were developed using the well-tuned PI controller. Images displayed in (d), (e), and (f) were generated using the PPF controller. A significant improvement in image quality can be observed. Third column: We were able to generate images at scan frequencies beyond the AFM limit of $30 \mathrm{~Hz} .40,50$, and $60 \mathrm{~Hz}$ scans are illustrated in (g), (h), and (i), respectively.

sample at identical frequencies but this time with the PPF control scheme implemented on the scanner. These images are plotted in Fig. 8 for comparison and illustrate a drastic improvement in image quality and sharpness. Furthermore, our modifications enabled us to scan beyond the $30 \mathrm{~Hz}$ speed set by the AFM standard software. In particular, we developed scans of the sample at 40,50, and $60 \mathrm{~Hz}$ with the same resolution. These results are also plotted in Fig. 8.

In order to further analyze the AFM images illustrated in Fig. 8, we plot the cross-section curves of these images at about $y=4 \mu \mathrm{m}$ in Fig. 9. The cross-section curves were taken in parallel to the square profile of the calibration grating. The scan direction for Figs. 9(a)-9(c) is from 0 to $8 \mu \mathrm{m}$ and the scan direction for Figs. $9(\mathrm{~d})-9(\mathrm{i})$ is from 8 to $0 \mu \mathrm{m}$. It can be observed that by using the PPF control scheme, the height-profile of the calibration grating was better captured. This controller enables us to set the feedback gain of the $z$ axis significantly higher than the well-tuned PI controller, resulting in a better scan. However, the accuracy of the curves representing the true height-profile of the calibration grating decreases with increasing scan frequency. At high scan frequencies, the $z$ axis feedback loop is simply not fast enough to detect changes in the height of the calibration grating. Nevertheless, it can be observed that the PPF control scheme results in better images even at high scan frequencies. 

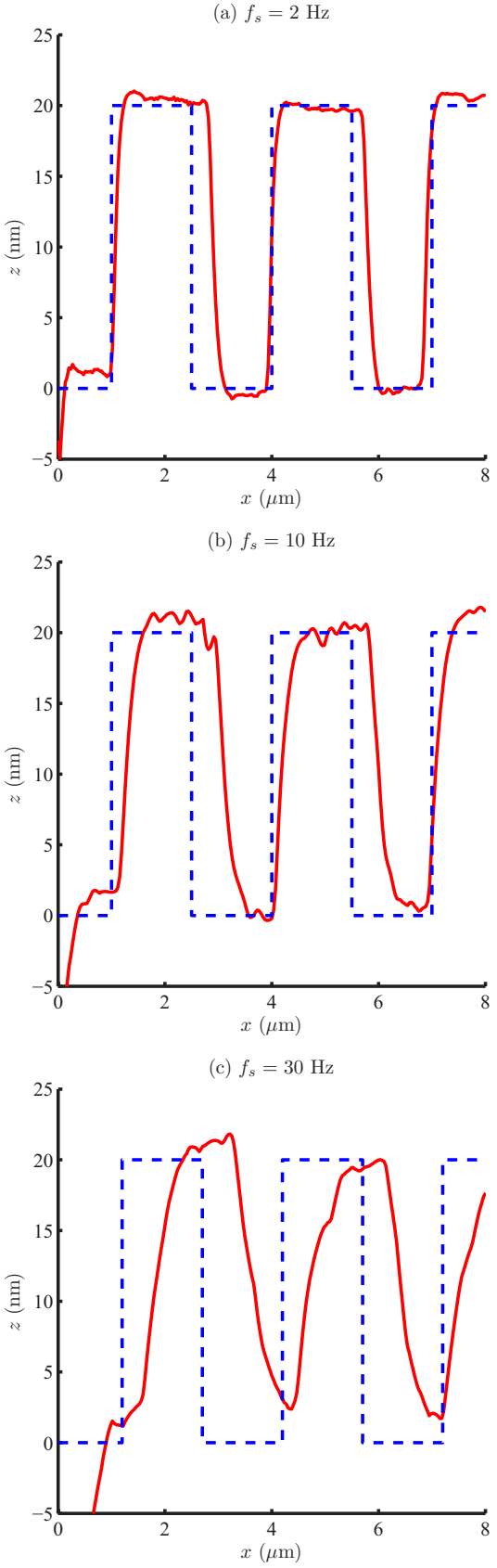

(d) $f_{s}=2 \mathrm{~Hz}$

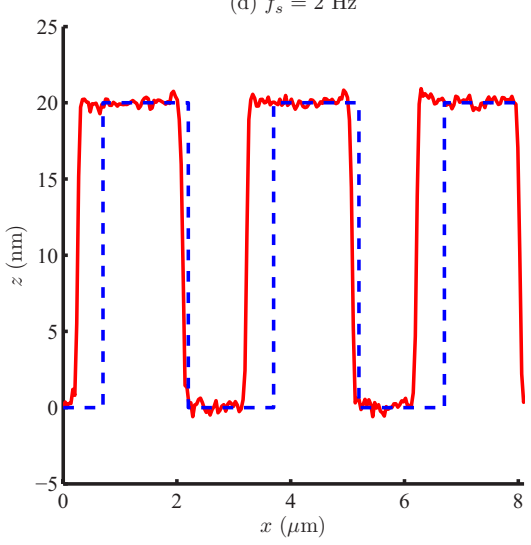

(e) $f_{s}=10 \mathrm{~Hz}$

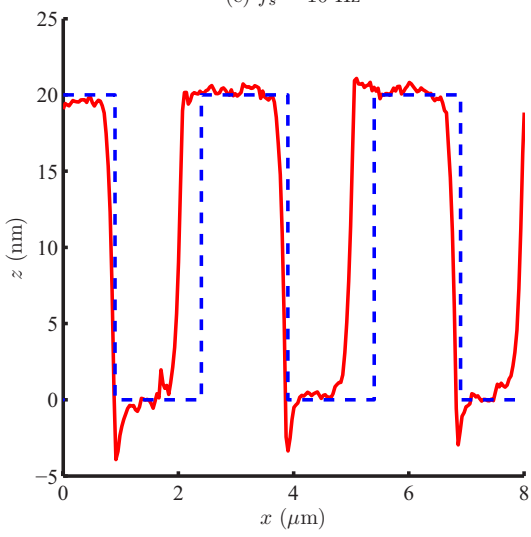

(f) $f_{s}=30 \mathrm{~Hz}$

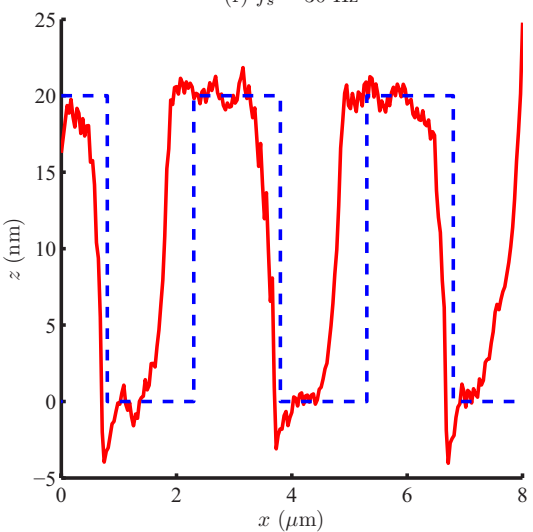

(g) $f_{s}=40 \mathrm{~Hz}$

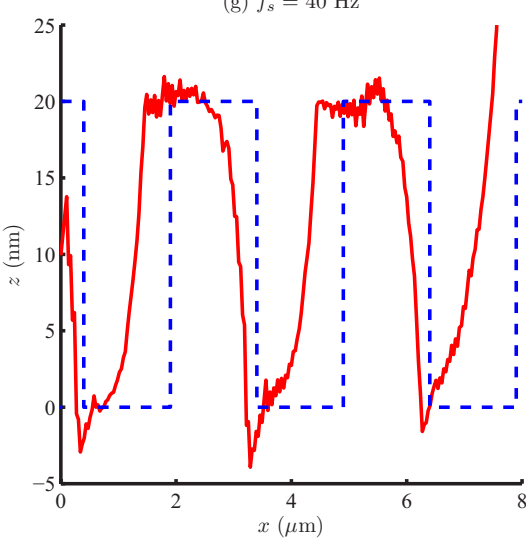

(h) $f_{s}=50 \mathrm{~Hz}$

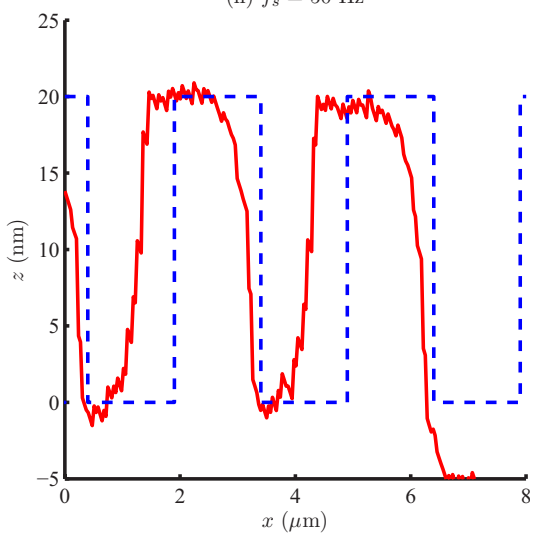

(i) $f_{s}=60 \mathrm{~Hz}$

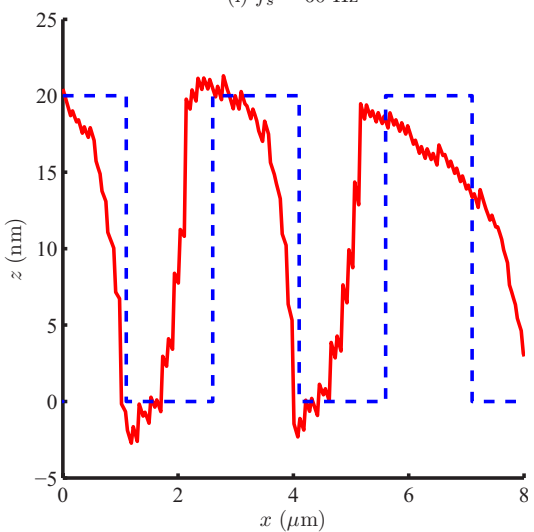

FIG. 9. (Color online) Cross-section (solid) and reference (dash) curves of the AFM images illustrated in Figs. 8(a)-8(i). The cross-section curves were taken about the center of the AFM images and parallel to the square profile of the calibration grating. The scan direction of the curves displayed in (a), (b), and (c) is from 0 to $8 \mu \mathrm{m}$. The scan direction of the curves displayed in (d) to (i) are from 8 to $0 \mu \mathrm{m}$.

\section{DISCUSSION AND CONCLUSIONS}

To this end, we wish to draw comparisons between the implemented PPF control scheme and other existing methods that were mentioned in the Introduction. The use of $H_{\infty}$ control for designing feedback controllers to improve the accuracy and speed of AFM scanners has been shown to be quite successful in Refs. 4 and 20. While these $H_{\infty}$ controllers provide adequate closed-loop robustness, they are often compensators of rather high orders. Thus, these controllers are more complex and their implementation may need a more sophisticated setup when compared with the proposed PPF controller, which has a very simple structure. In our work, the implementation of the PPF control scheme has resulted in a high closed-loop bandwidth of about $300 \mathrm{~Hz}$ and a damping of more than $30 \mathrm{~dB}$ at the scanner's first resonant mode. These improvements were achieved with a third order controller that is very straight forward to implement using either analog or digital methods.

In Refs. 18 and 22, a feedfoward input was used to compensate for vibration in feedback controlled AFM piezoactuator systems. This method can be quite effective in compensating induced structural vibration as proved experimentally in both Refs. 22 and 18. However, it may not be as effective against external vibrations and noise since the piezoactuators are still highly resonant structures. In our work, the overall mechanical damping of the piezoelectric 
tube was increased by using the PPF controllers. This makes the piezoelectric tube impervious against the induced and the external vibrations. Furthermore, if needed a feedfoward controller can be added to our controller to enable faster scans.

In conclusion, we demonstrated that by augmenting the damping of an AFM scanner using a PPF controller and minimizing the cross coupling between its fast and slow axes by incorporating an additional integral controller, and also by using charge drive on its fast axis, the quality of the developed image could be drastically improved. This is a direct result of the improvement in lateral positioning of the AFM scanner. We were also able to scan at frequencies beyond the limit set by the AFM software. Future research will involve minimizing cross coupling to the vertical axis of the scanner to achieve higher resolution images at faster scans.

${ }^{1}$ G. Binnig, C. F. Quate, and C. Gerber, Phys. Rev. Lett. 56, 930 (1986).

${ }^{2}$ E. Meyer, H. J. Hug, and R. Bennewitz, Scanning Probe Microscopy (Springer, Heidelberg, 2004).

${ }^{3}$ G. Binnig and D. P. E. Smith, Rev. Sci. Instrum. 57, 1688 (1986).

${ }^{4}$ S. Salapaka, A. Sebastian, J. P. Cleveland, and M. V. Salapaka, Rev. Sci. Instrum. 73, 3232 (2002).

${ }^{5}$ A. Sebastian and S. M. Salapaka, IEEE Trans. Control Syst. Technol. 13, 868 (2005).

${ }^{6}$ S. Aphale, B. Bhikkaji, and S. O. R. Moheimani, IEEE Trans. Nanotechnol. 7, 79 (2008).

${ }^{7}$ P. Ge and M. Jouaneh, Precis. Eng. 17, 211 (1995).

${ }^{8}$ R. B. Mrad and H. Hu, IEEE/ASME Trans. Mechatron. 7, 479 (2002).

${ }^{9}$ S. H. Lee and T. J. Royston, J. Acoust. Soc. Am. 108, 2843 (2000).

${ }^{10}$ T. Fett and G. Thun, J. Mater. Sci. Lett. 17, 1929 (1998).

${ }^{11}$ R. S. Robinson, J. Comput.-Assist. Microsc. 2, 53 (1996).

${ }^{12}$ H. Jung, J. Y. Shim, and D. Gweon, Rev. Sci. Instrum. 71, 3436 (2000).

${ }^{13}$ S. Salapaka, A. Sebastian, J. P. Cleveland, and M. V. Salappaka, Proceedings of the American Control Conference, 2002 (unpublished), pp. 19661971.

${ }^{14}$ D. Croft, G. Shed, and S. Devasia, ASME J. Dyn. Syst., Meas., Control 123, 35 (2001).

${ }^{15}$ B. Mokaberi and A. G. Requicha, IEEE Trans. Autom. Sci. Eng. 3, 199
(2006).

${ }^{16}$ G. Schitter and M. J. Rost, Mater. Today 11, 40 (2008).

${ }^{17}$ B. P. Lathi, Linear Systems and Signals, 2nd ed. (Oxford University Press, New York, 2004).

${ }^{18}$ Y. Li and J. Bechhoefer, Rev. Sci. Instrum. 78, 013702 (2007).

${ }^{19}$ X. Tian, N. Xi, Y. Wang, Z. Dong, and W. Li, International Conference on Control Automation, Robotics, and Vision, 2004 (unpublished), pp. 15811586.

${ }^{20}$ N. Tamer and M. Dahleh, Proceedings on the 33rd Conference Decision Control, 1994 (unpublished), pp. 1826-1830.

${ }^{21}$ A. Daniele, S. Salapaka, M. V. Salapaka, and M. Dahleh, Proceedings on the American Control Conference, 1999 (unpublished), pp. 253-257.

${ }^{22}$ K. K. Leang and S. Devasia, IEEE Trans. Control Syst. Technol. 15, 927 (2007).

${ }^{23}$ B. Bhikkaji, M. Ratnam, A. J. Fleming, and S. O. R. Moheimani, IEEE Trans. Control Syst. Technol. 5, 853 (2007).

${ }^{24}$ W. Zhang, L. Miao, Y. Zheng, Z. Dog, and N. Xi, Proceedings on the Third IEEE International Conference on Nano/Micro Engineered and Molecular Systems, 2008 (unpublished), pp. 617-621.

${ }^{25}$ S. Devasia, E. Eleftheriou, and S. O. R. Moheimani, IEEE Trans. Control Syst. Technol. 15, 802 (2007).

${ }^{26}$ A. J. Fleming and S. O. R. Moheimani, IEEE Trans. Control Syst. Technol. 14, 33 (2006)

${ }^{27}$ A. J. Fleming and K. K. Leang, Ultramicroscopy 108, 1551 (2008).

${ }^{28}$ T. McKelvey, H. Ackay, and L. Ljung, IEEE Trans. Autom. Control 41, 960 (1996).

${ }^{29}$ T. McKelvey, A. J. Fleming, and S. O. R. Moheimani, Trans. ASME, J. Vib. Acoust. 124, 414 (2002).

${ }^{30}$ R. Comstock, U.S. Patent No. 4,263,527 (21 April 1981).

${ }^{31}$ C. Newcomb and I. Flinn, Electron. Lett. 18, 442 (1982).

${ }^{32}$ A. J. Fleming and S. O. R. Moheimani, Electron. Lett. 39, 282 (2003).

${ }^{33}$ A. J. Fleming and S. O. R. Moheimani, J. Intell. Mater. Syst. Struct. 15, 77 (2004).

${ }^{34}$ A. J. Fleming and S. O. R. Moheimani, Rev. Sci. Instrum. 76, 073707 (2005).

${ }^{35}$ J. L. Fanson and T. K. Caughey, AIAA J. 28, 717 (1990).

${ }^{36}$ K. H. Rew, J. H. Han, and I. Lee, J. Intell. Mater. Syst. Struct. 13, 13 (2002).

${ }^{37}$ G. Song, S. P. Schmidt, and B. N. Agrawal, J. Guid. Control Dyn. 25, 179 (2002).

${ }^{38}$ S. O. R. Moheimani, B. J. G. Vautier, and B. Bhikkaji, IEEE Trans. Control Syst. Technol. 14, 443 (2006). 